\title{
A COUNTEREXAMPLE TO A PROBLEM ON POINTS OF CONTINUITY IN BANACH SPACES
}

\author{
N. GHOUSSOUB, B. MAUREY AND W. SCHACHERMAYER
}

\begin{abstract}
In a previous paper of the first two authors [GM] the space $J T_{\infty}$ was constructed as a James space over a tree with infinitely many branching points. It was proved that the predual $B_{\infty}$ of $J T_{\infty}$ fails the "point of continuity property."

In the present paper we show that $B_{\infty}$ has the so-called "convex point of continuity property" thus answering a question of Edgar and Wheeler [EW] in the negative.
\end{abstract}

1. Definitions, notations and preliminaries. Recall $[\mathbf{B R}]$ that a Banach space $X$ has the point of continuity property (PCP) if for every weakly closed bounded subset $C$ of $X$ there is $x \in C$ such that the weak and norm topologyrestricted to $C$-coincide at $x$. An equivalent formulation goes as follows (compare [B, Proposition 1]): For every bounded subset $C$ of $X$ and for $\varepsilon>0$ there is a relatively weakly open subset $U$ of $C$ such that the norm-diameter of $U$ is less than $\varepsilon$.

Previously, J. Bourgain [B] introduced (under the name of property $(*)$ ) the following weaker concept: A Banach space $X$ has the convex point of continuity property (CPCP) if every closed convex bounded subset $C$ of $X$ has a point $x$ where the relative weak and norm topologies coincide. Again this may be rephrased as follows: For every convex bounded subset $C$ of $X$ and $\varepsilon>0$ there is a relatively weakly open subset $U$ of $C$ of norm-diameter less than $\varepsilon$. The question whether PCP and CPCP are in fact equivalent remained open and was explicitly asked in [EW]. We shall show that the space $B_{\infty}$ furnishes an example with CPCP but failing PCP.

Recall from $[\mathbf{G M}]$ the definition of $J T_{\infty}$ : We consider the tree with infinitely many branching points

$$
T_{\infty}=\sum_{k=0}^{\infty} \mathbf{N}^{k} .
$$

If $t=\left(n_{1}, \ldots, n_{k}\right) \in T_{\infty}$, set $|t|=k$ to be the rank of $t$. If $x=\left(x_{t}\right)_{t \in T_{\infty}}$ is a real-valued function of finite support defined on $T_{\infty}$, let

$$
\|x\|_{J T_{\infty}}=\sup \left(\sum_{i=1}^{n}\left(\sum_{t \in S_{i}} x_{t}\right)^{2}\right)^{1 / 2}
$$

the supremum taken over all families $\left(S_{1}, \ldots, S_{n}\right)$ of disjoint segments in $T_{\infty} . J T_{\infty}$ will be the completion of this normed space.

Received by the editors December 25, 1985.

1980 Mathematics Subject Classification (1985 Revision). Primary 46B20, 46B22, 46 G10. 
As the finite vectors are dense in $J T_{\infty}$, an element $y \in J T_{\infty}^{*}$ is characterized by its values $y_{t}=y\left(e_{t}\right)$ on the unit vectors $e_{t}, t \in T_{\infty}$. The space $B_{\infty}$ will be the norm closure of the span of the coefficient functionals-again denoted $\left\{e_{t}, t \in T_{\infty}\right\}$-in $J T_{\infty}^{*}$.

We shall say that a subset $A \subseteq T_{\infty}$ is full, if for each segment $S$, the intersection $S \cap A$ is again a segment. In this case, the projection

$$
P_{A}: J T_{\infty} \rightarrow J T_{\infty}, \quad \sum_{t \in T_{\infty}} x_{t} e_{t} \rightarrow \sum_{t \in A} x_{t} e_{t}
$$

is a contraction. The adjoint of $P_{A}$-still denoted $P_{A}$-defines a contraction from $B_{\infty}$ to $B_{\infty}$.

We shall need the following cases of full sets $A \subseteq T_{\infty}$ : If $\gamma$ is a branch in $T_{\infty}$, we denote by $P_{\gamma}$ the projection defined by the set $\gamma . L_{n}$ will denote the $n$th line of $T_{\infty}$, i.e. $L_{n}:\{t:|t|=n\}$ and $P_{n}$ the projection defined by $L_{n}$. If $n \leq m$ we denote $P_{n}^{m}$ the projection defined by $L_{n} \cup L_{n+1} \cup \cdots \cup L_{m}$.

For each branch $\gamma=\left\{\phi,\left(n_{1}\right),\left(n_{1}, n_{2}\right), \ldots,\left(n_{1}, n_{2}, \ldots, n_{k}\right), \ldots\right\}$ in $T_{\infty}$, define

$$
S_{\gamma}: J T_{\infty}^{*} \rightarrow \mathbf{R}, \quad y \rightarrow \lim _{t \in \gamma} y_{t}
$$

which is well defined as $P_{\gamma}\left(J T_{\infty}\right)$ is isometric to the usual James space $J$. The collection of branches $\gamma$ may be identified-in an obvious way-with $\mathbf{N}^{\mathbf{N}}$. The following lemma is just a slight variant of Theorem 1 of $[\mathbf{L S}]$ and the proof carries over almost verbatim.

\subsection{LEMMA. The operator}

$$
S: J T_{\infty}^{*} \rightarrow l^{2}\left(\mathbf{N}^{\mathbf{N}}\right), \quad y \rightarrow\left(\lim _{t \in \gamma} y_{t}\right)_{\gamma \in \mathbf{N}^{\mathbf{N}}}
$$

is a well-defined quotient map and the kernel of $S$ equals $B_{\infty}$.

Note the following consequence of Lemma 1.1 which we shall use in the sequel:

1.2 LEMMA. Let $y \in J T_{\infty}^{*}$ such that

$$
\liminf _{n \rightarrow \infty}\left\|P_{n}(y)\right\|_{\infty}=0
$$

where $\|\cdot\|_{\infty}$ denotes the sup-norm for a function defined on $T_{\infty}$. Then $y$ is an element of $B_{\infty}$. In particular, if $(*)$ holds true, there cannot be an increasing sequence $\left(n_{k}\right)_{k=1}^{\infty}$ and $\alpha>0$ such that

$$
\left\|P_{n_{k}+1}^{n_{k+1}}(y)\right\|_{J T_{\infty}^{*}} \geq \alpha, \quad k=1,2, \ldots
$$

ProOF. Condition (*) implies that for each branch $\gamma$ the limit $S_{\gamma}(y)$ equals 0 ; hence $S(y)=0$ and by Lemma 1.1 we infer that $y \in B_{\infty}$.

For the second part, note that $B_{\infty}$ is spanned by the coefficient functionals $e_{t}$, $t \in T_{\infty}$, hence $(* *)$ is contradictory to $(*)$.

2. The main result. The following lemma is crucial for the proof of Theorem 2.2. The proof is an interplay of a gliding hump argument with the formation of Cesàro means. 
2.1 LEMMA. Let $C$ be a convex, bounded subset of $B_{\infty}, M \in \mathbf{N}$, and $\varepsilon>0$. There is a relatively weakly open convex subset $U$ of $C$ and $N>M$ such that

$$
\left\|P_{N}(U)\right\|_{\infty}=\sup \left\{\left\|P_{N}(y)\right\|_{\infty}: y \in U\right\} \leq \varepsilon .
$$

ProOF. We may and do suppose that $C$ is contained in the unit ball of $B_{\infty}$ and that $0<\varepsilon<1$. Choose natural numbers $n$ and $m$ such that $n>2 / \varepsilon$ and $m>\left(8 n / \varepsilon^{2}\right)+1$ and choose $\eta>0$ such that $\eta<\varepsilon / 2 m n$. Fix an element $y_{0}^{1}$ in $C$ and choose $N>M$ such that $\left\|P_{N}\left(y_{0}^{1}\right)\right\|_{\infty}<\eta$. Suppose the lemma is not true (for the $N$ chosen above). We shall obtain a contradiction by double-induction: For $0 \leq i \leq n$ and $1 \leq j \leq m$ we shall find $y_{i}^{j} \in C$ and, for $1 \leq i \leq n$ and $1 \leq j \leq m$, $t_{i}^{j} \in L_{N}$ such that

$$
\begin{aligned}
& \left|y_{i}^{j}\left(t_{i}^{j}\right)\right|>\varepsilon \\
& y_{0}^{j}=n^{-1}\left(y_{1}^{j-1}+\cdots+y_{n}^{j-1}\right) \\
& \left|\left(y_{i}^{j}-y_{0}^{j}\right)\left(t_{p}^{q}\right)\right|<\eta \\
& \left|y_{i}^{j}\left(t_{p}^{q}\right)\right|<\eta
\end{aligned}
$$

$$
1 \leq j \leq m, 1 \leq i \leq n
$$$$
2 \leq j \leq m \text {, }
$$$$
\text { if }(q, p)<(j, i) \text { lexicographically, }
$$$$
\text { i.e. } q<j \text { or } q=j \text { and } p<i \text {, }
$$$$
\text { if }(q, p)>(j, i) \text { lexicographically. }
$$

Let us suppose for the moment that we have done the construction and finish the proof. Fix $(q, p)$ with $1 \leq q<m$ and $1 \leq p \leq n$. Note that (ii) and (iv) imply $\left|y_{0}^{q}\left(t_{p}^{q}\right)\right|<\eta$. Now apply (i) (for $(j, i)$ equal to $(q, p)$ ), (ii), (iii), and (iv) to obtain

$$
\left|y_{0}^{q+1}\left(t_{p}^{q}\right)\right|>\varepsilon / n-2 \eta \text {. }
$$

Repeated application of (ii) and (iii) shows

$$
\left|y_{0}^{m}\left(t_{p}^{q}\right)\right|>\varepsilon / n-(m-q+1) \eta \geq \varepsilon / n-m \eta \geq \varepsilon / 2 n .
$$

Hence

$$
\begin{aligned}
\left\|y_{0}^{m}\right\|_{B_{\infty}}^{2} \geq\left\|P_{N}\left(y_{0}^{m}\right)\right\|_{B_{\infty}}^{2} & \geq \sum_{q=1}^{m-1} \sum_{p=1}^{n}\left|y_{0}^{m}\left(t_{p}^{q}\right)\right|^{2} \\
& \geq(m-1) \cdot n \cdot(\varepsilon / 2 n)^{2} \geq 2
\end{aligned}
$$

which contradicts the assumption that $C$ is contained in the unit ball of $B_{\infty}$.

So, let us do the inductive construction. We have already chosen $y_{0}^{1}$; let $C_{0}^{1}=C$. By assumption there is $y_{1}^{1} \in C_{0}^{1}$ and $t_{1}^{1} \in L_{N}$ s.t. $\left|y_{1}^{1}\left(t_{1}^{1}\right)\right|>\varepsilon$. Let

$$
A_{1}^{1}=\left\{t \in L_{N}:\left|y_{1}^{1}(t)\right|>\eta\right\}
$$

and

$$
C_{1}^{1}=\left\{y \in C_{0}^{1}:\left|\left(y-y_{0}^{1}\right)(t)\right|<\eta \text { for } t \in A_{1}^{1}\right\} .
$$

Clearly $C_{1}^{1}$ is a relatively weakly open convex subset of $C$; hence by assumption there is $y_{2}^{1} \in C_{1}^{1}$ and $t_{2}^{1} \in L_{N}$ s.t. $\left|y_{2}^{1}\left(t_{2}^{1}\right)\right|>\varepsilon$. Let

$$
A_{2}^{1}=A_{1}^{1} \cup\left\{t \in L_{N}:\left|y_{2}^{1}(t)\right|>\eta\right\}
$$

and

$$
C_{2}^{1}=\left\{y \in C_{0}^{1}:\left|\left(y-y_{0}^{1}\right)(t)\right|<\eta \text { for } t \in A_{2}^{1}\right\} .
$$

Continue in an obvious way to find $y_{1}^{1}, \ldots, y_{n}^{1}$ and $t_{1}^{1}, \ldots, t_{n}^{1}$ satisfying (i), (iii), and (iv). Define

$$
y_{0}^{2}=n^{-1}\left(y_{1}^{1}+\cdots+y_{n}^{1}\right), \quad A_{0}^{2}=A_{n}^{1},
$$


and

$$
C_{0}^{2}=\left\{y \in C_{0}^{1}:\left|\left(y-y_{0}^{2}\right)(t)\right|<\eta \text { for } t \in A_{0}^{2}\right\}
$$

Again $C_{0}^{2}$ is a relatively weakly open, nonempty, and convex subset of $C$; hence we may find $y_{1}^{2} \in C_{0}^{2}$ and $t_{1}^{2} \in L_{N}$ such that $\left|y_{1}^{2}\left(t_{1}^{2}\right)\right|>\varepsilon$.

Note that $t_{1}^{2}$ cannot belong to $A_{0}^{2}$ as the elements of $C_{0}^{2}$ are smaller than $2 \eta+n^{-1}$ on the elements of $A_{0}^{2}$. Hence (iv) is satisfied for $(q, p)=(2,1)$ and $(i, j)<(q, p)$. Let

and

$$
A_{1}^{2}=A_{0}^{2} \cup\left\{t \in L_{N}:\left|y_{1}^{2}(t)\right|>\eta\right\}
$$

$$
C_{1}^{2}=\left\{y \in C_{0}^{2}:\left|\left(y-y_{0}^{2}\right)(t)\right|<\eta \text { for } t \in A_{1}^{2}\right\} \text {. }
$$

Again by assumption there is $y_{2}^{2} \in C_{1}^{2}$ and $t_{2}^{2} \in L_{N}$ s.t. $\left|y_{2}^{2}\left(t_{2}^{2}\right)\right|>\varepsilon$, etc.; we thus find $y_{1}^{2}, \ldots, y_{n}^{2}$ and $t_{1}^{2}, \ldots, t_{n}^{2}$ satisfying (i), (iii), and (iv). Now define

$$
\begin{aligned}
y_{0}^{3} & =n^{-1}\left(y_{1}^{2}+\cdots+y_{n}^{2}\right), \quad A_{0}^{3}=A_{n}^{2}, \\
C_{0}^{3} & =\left\{y \in C_{0}^{2}:\left|\left(y-y_{0}^{3}\right)(t)\right|<\eta \text { for } t \in A_{0}^{3}\right\} .
\end{aligned}
$$

Continue in an obvious way for $j=1, \ldots, m$ to finish the inductive procedure and the proof of the lemma.

\subsection{ThEOREM. $B_{\infty}$ has $C P C P$.}

PROOF. If the theorem were false we could find a convex bounded $C \subseteq B_{\infty}$ and $\alpha>0$ such that each relatively weakly open subset $U$ of $C$ has norm-diameter greater than $3 \alpha$.

Again we shall argue inductively: Let $y_{1} \in C,\left\|y_{1}\right\|>\alpha$ and find $x_{1} \in J T_{\infty}$, $\left\|x_{1}\right\|=1$ of finite support, say $\operatorname{supp}\left(x_{1}\right) \subseteq L_{1} \cup \cdots \cup L_{M_{1}}$ s.t. $\left\langle x_{1}, y_{1}\right\rangle>\alpha$. Let

$$
C_{1}=\left\{y \in C:\left\langle x_{1}, y\right\rangle>\alpha\right\}
$$

and apply Lemma 2.1 to $C_{1}$ to find $N_{1}>M_{1}$ and a relatively weakly open, nonempty, and convex $D_{1} \subseteq C_{1}$ s.t. $\left\|P_{N}\left(D_{1}\right)\right\|_{\infty}<1$. Finally note that $P_{1}^{N_{1}}\left(B_{\infty}\right)$ is isomorphic to $l^{2}$, which has CPCP, hence we may find a relatively weakly open, nonempty, convex $E_{1} \subseteq D_{1}$ s.t.

$$
\operatorname{diam}\left(P_{1}^{N_{1}}\left(E_{1}\right)\right)<\alpha
$$

hence

$$
\operatorname{diam}\left(P_{N_{1}+1}^{\infty}\left(E_{1}\right)\right)>2 \alpha .
$$

Find $y_{2} \in E_{1}$ and a finitely valued $x_{2} \in J T_{\infty}$ with the support contained in $L_{N_{1}+1} \cup \cdots \cup L_{M_{2}},\left\|x_{2}\right\|=1$, s.t. $\left\langle x_{2}, y_{2}\right\rangle>\alpha$ and let

$$
C_{2}=\left\{y \in E_{1}:\left\langle x_{2}, y\right\rangle>\alpha\right\} \text {. }
$$

Apply Lemma 2.1 to find $N_{2}>M_{2}$ and $D_{2} \subseteq C_{2}$, s.t. $\left\|P_{N_{2}}\left(D_{2}\right)\right\|_{\infty}<1 / 2$. Finally find $E_{2} \subseteq D_{2}$ s.t. $\operatorname{diam}\left(P_{1}^{N_{2}}\left(E_{2}\right)\right)<\alpha$; hence $\operatorname{diam}\left(P_{N_{2}+1}^{\infty}\left(E_{2}\right)\right)>2 \alpha$.

Continue in an obvious way to find $C_{n} \supset D_{n} \supset E_{n} \supset C_{n+1} \supset \cdots, M_{n}<N_{n}<$ $M_{n+1}<N_{n+1}<\cdots$, and $x_{n} \in J T_{\infty},\left\|x_{n}\right\|=1$ with $\operatorname{supp}\left(x_{n}\right) \subseteq L_{N_{n-1}+1} \cup \cdots \cup$ $L_{M_{n}}$ s.t.

while

$$
C_{m} \subseteq\left\{y \in B_{\infty}:\left\langle x_{n}, y\right\rangle>\alpha\right\}, \quad m>n
$$

$$
\left\|P_{N_{n}}\left(C_{m}\right)\right\|_{\infty}<n^{-1}, \quad m>n
$$


Let $\bar{C}_{n}^{*}$ be the $\sigma\left(J T_{\infty}^{*}, J T_{\infty}\right)$-closure of $C_{n}$. By weak-star compactness there is an element $y \in J T_{\infty}^{*}$,

$$
y \in \bigcap_{n=1}^{\infty} \bar{C}_{n}^{*} .
$$

This $y$ has the (impossible) properties described in Lemma 1.2, so we arrive at a contradiction and prove the theorem.

\section{REFERENCES}

[BR] J. Bourgain and H. P. Rosenthal, Applications of the theory of semi-embeddings to Banach space theory, J. Funct. Anal. 52 (1983), 149-188.

[B] J. Bourgain, Dentability and finite dimensional decompositions, Studia Math. 67 (1980), 135-148.

[EW] G. A. Edgar and R. F. Wheeler, Topological properties of Banach spaces, Pacific J. Math. 115 (1984), 317-350.

[GM] N. Ghoussoub and B. Maurey, $G_{\delta}$-embeddings in Hilbert space, J. Funct. Anal. 61 (1985), 72-97.

[LS] J. Lindenstrauss and C. Stegall, Examples of separable spaces which do not contain l $^{1}$ and whose duals are not separable, Studia Math. 54 (1975), 81-105.

Department of Mathematics, The University of British Columbia, VancouVER, B.C., CANADA V6T 1T4

Département de Mathématiques, Université Paris VII, Paris, France

INSTITUT FÜR MATHEMATIK, JOHANNES KEPLER UNIVERSITÄT, A-4040 LINZ, AUSTRIA 